Tropical Journal of Pharmaceutical Research April 2018; 17 (4): 669-673

ISSN: $1596-5996$ (print); 1596-9827 (electronic)

(C) Pharmacotherapy Group, Faculty of Pharmacy, University of Benin, Benin City, 300001 Nigeria.

\title{
Galantamine protects against hydrochloric acid aspiration- induced acute respiratory distress syndrome in rabbits
}

\author{
Yi Yang ${ }^{1}$, Yan Peng ${ }^{2}$, Jin Yang ${ }^{1^{*}}$ \\ ${ }^{1}$ Department of Intensive Care Unit, Chongqing People's Hospital, 400014,Chongqing,China, ${ }^{2}$ Department of Intensive Care \\ Unit, First Affiliated Hospital of Chongqing Medical University, 400016, Chongqing, China
}

*For correspondence: Email: cb0750@163.com; Tel: +86-023-6351524

Sent for review: 5 February 2018

Revised accepted: 28 March 2018

\begin{abstract}
Purpose: To study the effect of galantamine on anti-inflammatory responses in acid aspiration-induced acute respiratory syndrome (ARDS), and the underlying mechanism.

Methods: Six groups of male rabbits (156), i.e., control group, ARDS group, galantamine + ARDS (GAL) group, galantamine + ARDS + methyllycaconitine (MLA) group, galantamine + ARDS + vagotomy (Vag) group, and galantamine $+A R D S+$ atropine sulfate (ATS) group. ARDS model was produced by acid aspiration. After $4 \mathrm{~h}, \mathrm{TNF}-\alpha, \mathrm{IL}-6$ and IL-1 $\beta$ were assayed in lung tissue, while corticosterone levels were determined in blood. Histopathological lesions and wet-to-dry (W/D) weight ratio of lung tissue $(n=$ 10) were assessed. After 12 h, HMGB1 protein and corticosterone levels were determined in lung tissue and blood, respectively. Mortality rate was determined after $72 h$.

Results: Acid aspiration-induced ARDS induced disorganization of lung structure, and elevated TNF- $\alpha$, $I L-6, I L-1 \beta$, and HMGB1activities, and lung W/D weight ratio. The acid-induced ARDS, as well as increases in W/D weight ratio, pro-inflammatory cytokines and lung lesions were significantly decreased by galantamine pretreatment. Methyllycaconitin, vagotomy and atropine sulfate abolished the galantamine-induced suppression of acute inflammatory response, pathological changes in lungs, and W/D weight ratio. However, serum corticosterone levels were not significantly altered in each group.

Conclusion: Galantamine reduces inflammation in acid aspiration-induced ARDS by the cholinergic anti-inflammatory pathway.
\end{abstract}

Keywords: Galantamine, Hydrochloric acid, Aspiration-induced, Respiratory distress syndrome, Methyllycaconitin, Vagotomy, Atropine sulfate

\begin{abstract}
This is an Open Access article that uses a funding model which does not charge readers or their institutions for access and distributed under the terms of the Creative Commons Attribution License (http://creativecommons.org/licenses/by/4.0) and the Budapest Open Access Initiative (http://www.budapestopenaccessinitiative.org/read), which permit unrestricted use, distribution, and reproduction in any medium, provided the original work is properly credited.
\end{abstract}

Tropical Journal of Pharmaceutical Research is indexed by Science Citation Index (SciSearch), Scopus, International Pharmaceutical Abstract, Chemical Abstracts, Embase, Index Copernicus, EBSCO, African Index Medicus, JournalSeek, Journal Citation Reports/Science Edition, Directory of Open Access Journals (DOAJ), African Journal Online, Bioline International, Open-J-Gate and Pharmacy Abstracts

\section{INTRODUCTION}

The etiology of acute respiratory distress syndrome (ARDS) is associated with damage to the membrane of capillaries of the alveoli which compromises their permeability and results in edema [1]. Aspiration pneumonitis is one of the important causes of ARDS [2]. Studies so far are yet to unravel the signal pathways implicated in the pathogenesis of ARDS. Nonetheless, research findings indicate that ARDS may be triggered on and amplified by cytokine mediation through enhanced expression of high mobility group box 1 (HMGB1), interleukin (IL)-1, IL-6, 
and tumor necrosis factor (TNF)- $\alpha$ in the lungs [3].

In situations such as autoimmune diseases and sepsis, the anti-inflammatory functions of the cholinergic nervous system results in blockage of uncontrolled expression of pro-inflammatory mediators, through the vagus nerve $[4,5]$. Vagal efferent stimulation can protect against Mesobuthus tamulus venom-induced ARDS through anti-inflammatory mechanism [6]. The regulatory influence of the efferent vagus nerve on expression of pro-inflammatory cytokines is mediated through the cholinergic antiinflammatory route which involves $\alpha 7 n A C h R-$ dependent signal cascade [7]. This antiinflammatory route is under the control of neuronal muscarinic mechanisms [8]. It has been demonstrated that galantamine (GAL), a negative effector of cholinesterase, mitigated lipopolysaccharide-induced inflammatory responses in animal models through the cholinergic route [9].

The present study investigated the protective effects of GAL against acid aspiration-induced ARDS, and the mechanism involved.

\section{EXPERIMENTAL}

\section{Animals}

Adult New Zealand white rabbits (male, weight range: 1.9 - $2.5 \mathrm{~kg}$ ) were subjected to pentobarbital anesthesia (3\%, $25 \mathrm{mg} / \mathrm{kg}$ body weight), followed by uninterrupted administration at the rates range of $5-10 \mathrm{mg} / \mathrm{kg}$ body weight $/ \mathrm{h}$. The rabbit muscles were relaxed by administration of $0.2 \mathrm{mg} / \mathrm{kg}$ body weight pipecuronium bromide. The rabbits were ventilated during tracheotomy in SIMV mode with the aid of a Drager Evita 2 Dura ventilator (Drager Medical AG \& Co., Germany), under the following settings: $6 \mathrm{ml} / \mathrm{kg}$ body weight VT; maintenance of $\mathrm{PaCO}_{2}$ within $35-45 \mathrm{mmHg}$ via $\mathrm{RR}$ adjustments; $\mathrm{FIO}_{2}=0.3$; inspiratory-toexpiratory ratio of $1: 1$, and $2 \mathrm{cmH}_{2} \mathrm{O}$ positive end-pressure.

Physiological saline infusion was maintained at a constant rate of $8 \mathrm{ml} / \mathrm{kg}$ body weight $/ \mathrm{h}$, to ensure that arterial pressure values were $>80 \mathrm{mmHg}$. No vasoactive drugs were applied during the study which was approved by the Animal Ethical Committee of The People's Hospital of Chongqing City (approval no. 2017040502), and implemented according to "Principles of Laboratory Animal Care" (NIH publication no. 85 - 23, revised 1985) [10].

\section{Reagents}

The reagents used and their sources were: $\mathrm{GAL}$ (EMD Biosciences Inc.,USA); $\alpha 7$ nicotinic receptor blocking agent i.e. methyllycaconitine (MLA) as well as corticosterone and cytokine assay kits ( $R$ \& D Systems, USA); atropine sulfate (ATS, Hefeng Pharmaceutical Co., China); rabbit anti-HMGB1 polyclonal antibody (Boster Biotechnology Company, China), and HMGBI kits (Boster Biotech. Company Ltd, USA). Western blotting antibodies were product of Pierce Biotechnology, USA).

\section{Vagotomy}

In each rabbit, the right cervical vagus nerve was exposed, ligated using a 4 - 0 silk suture, and parted.

\section{Preparation of ARDS model}

Intra-tracheal instillation of $10^{-1} \mathrm{M} \mathrm{HCL}$ was done in the two lateral positions $(1.5 \mathrm{ml} / \mathrm{kg}$ per side) prior to a pause for about $5 \mathrm{sec}$ at a peak pressure of $25 \mathrm{cmH}_{2} \mathrm{O}$. The ARDS model was considered stable 30 min later if $\mathrm{PaO}_{2} / \mathrm{FIO}_{2}$ was less than 200, otherwise, the procedure was repeated until $\mathrm{PaO}_{2} / \mathrm{FIO}_{2}$ reached the predefined standard level.

\section{Experimental protocols}

Rabbits were observed for $30 \mathrm{~min}$ for stabilization after cannulation, and were then randomized into six groups (26 rabbits/group). Group 1: ARDS group; group 2 (ARDS + GAL group), in which GAL (4 mg/kg) was given i.p. 30 min prior to hydrochloric acid aspiration; group 3 (ARDS + GAL + MLA), in which MLA (4 mg/kg, i.p.) was administered 15 min before GAL; group 4 (ARDS + GAL + Vag) in which vagotomy was done 15 min before intraperitoneal GAL; group 5 (ARDS + GAL + ATS), in which ATS (4 mg/kg, i.p.) was administered $15 \mathrm{~min}$ before intraperitoneal GAL; group 6 (control), given in place of treatment, equivalent volume of saline intra-tracheally.

\section{Survival study}

The population of rabbits that survived in the six groups were noted $72 \mathrm{~h}$ after the hydrochloric acid aspiration.

\section{Histological analysis}

Four (4) h after hydrochloric acid aspiration, the rabbits were euthanized $(n=10)$. Lung tissue samples were processed for light microscopy in 
accordance with standard procedures [11]. Histological changes were scored by a pathologist naïve to the animal treatments, on a 5-point scale based on presence of, and extent of lesions such as edema, hemorrhage, and neutrophils: absent $=0$, mild $=1$, moderate $=2$, severe $=3$, and $4=$ overwhelming, with a maximum score of 12 points [12].

\section{Wet-to-dry weight ratio}

Following a 4-h euthanization, the right lungs were ligated, excised and washed with phosphate buffered saline (PBS). They were then blotted and weighed (wet weights). Thereafter, the samples were oven-dried to constant weights for $48 \mathrm{~h}$ at a temperature of 80 ${ }^{\circ} \mathrm{C}$ (dry weights). These measurements allowed for determination of the ratio of wet-to-dry (W/D).

\section{Corticosterone assay}

Blood was taken from the euthanized rabbits through cardiac puncture at 4 and $12 \mathrm{~h}$, and used for assay of corticosterone levels in serum in line with instructions contained in corticosterone assay kits.

\section{Cytokine assay}

Lung tissue homogenate was prepared at $\mathrm{pH} 7.2$ in PBS treated with protease inhibitor, using lung samples obtained $4 \mathrm{~h}$ after hydrochloric acid aspiration, rinsed of blood, and homogenized using Polytron (Brinkman) in homogenization buffer (PBS, containing a protease inhibitor cocktail, $\mathrm{pH} 7.2)(\mathrm{n}=10)$. The homogenates were clarified by centrifugation, and the resultant supernatants were subjected to ELISA assays for TNF- $\alpha, I L-1 \beta$ and IL- 6 . The protein contents of the supernatants were estimated using the Bradford procedure.

\section{Western blot analysis for HMGB1}

Lung tissue protein extracts prepared from samples taken after $12 \mathrm{~h}$ of $\mathrm{HCL}$ exposure were subjected to Western blot analysis in line with standard procedures. Following sequential incubation of the membrane with the respective primary and secondary antibodies, the membranes were washed thrice in TTBS buffer and visualized through chemiluminescence.

\section{Statistical analysis}

All data are presented mean \pm standard deviation (SD. Comparisons between different groups were done with analysis of variance (ANOVA) and Student-Newman-Keuls q-test. Values of $p<$
0.05 were taken as indicative of statistically significant differences.

\section{RESULTS}

\section{Mortality}

There was no mortality in the control group. However, mortalities in ARDS, ARDS + GAL, MLA, and ATS groups were 87.5, 12.5, 50 and $50 \%$, respectively.

\section{Pathological changes in lung tissue}

No lesions were evident in the photomicrograph of lung tissue from the control group. On the other hand, $\mathrm{HCL}$ aspiration for $4 \mathrm{~h}$ was associated with clear evidence of histopathological changes such as inflammation, hemorrhage, thickened alveolar walls, and pulmonary edema. However, GAL pretreatment led to significant reductions in the $\mathrm{HCL}$-induced pulmonary lesions. Methyllycaconitine, vagotomy and atropine sulfate reduced GAL-induced reduction in lung lesions and inflammation. These results are shown in Figures $1 \mathrm{~A}-1 \mathrm{~F}$. The degree of pulmonary damage was significantly higher in ARDS rabbits than in the GAL group $(p<0.05)$. Rabbits in the MLA, Vag and ATS groups had significantly higher aggregate scores in lung lesions, when compared with corresponding scores for $\mathrm{GAL}$ group ( $p<0.05$, Figure $1 \mathrm{G}$ ).
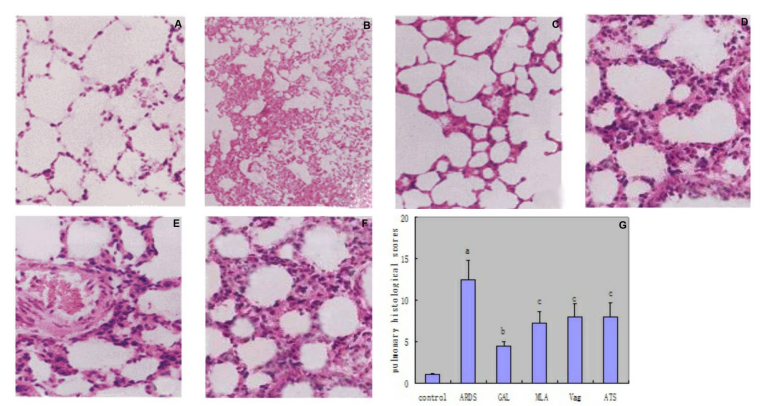

Figure 1: Photomicrographs of lung tissues from the 3 groups, and scores for pulmonary lesions. A: Control group; B: ARDS group; C: GAL group; D: MLA group; E: Vag group; F: ATS group (H \& E, x 200). G: scores for pulmonary lesions in the six groups. ${ }^{a} p<0.05$, ARDS group compared to control group; ${ }^{b} \underline{p}<0.05, \mathrm{GAL}$ group compared to ARDS group (ANOVA); ${ }^{c} p<0.05$, MLA, Vag, ATS groups compared to GAL group

\section{Influence of GAL on W/D index}

The W/D ratio of the control group was significantly lower than that of the ARDS $(p<$ 0.05). However, the GAL treatment led to 
significant decrease in $W / D$ relative to the ARDS group $(p<0.05)$. The $W / D$ ratios in the MLA, Vag and ATS groups were significantly higher than that of the GAL group, indicating that methyllycaconitine, vagotomy and atropine sulfate reduced GAL-induced attenuation of the degree of pulmonary edema in ARDS ( $p<0.05$, Figure 2$)$.

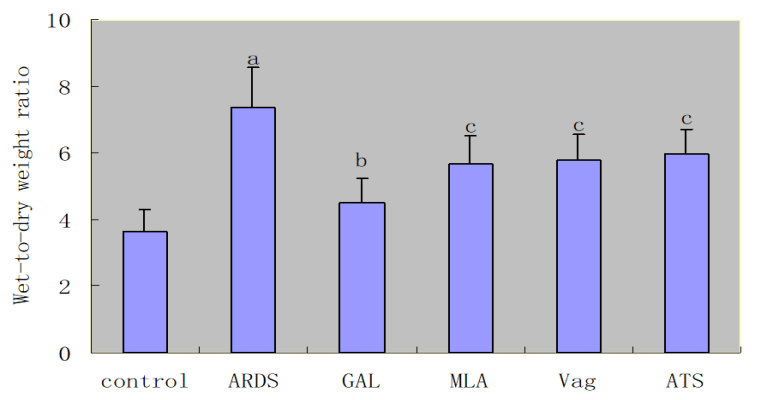

Figure 2: W/D ratios in the 6 groups. ${ }^{a} p<0.05$, ARDS group compared to control group; ${ }^{b} p<0.05$, GAL group compared to ARDS group; ${ }^{c} p<0.05$, MLA, Vag and ATS groups compared to GAL group. Values are mean $\pm S D$

\section{GAL suppressed HMGB1 expression in lungs}

HMGBI was expressed at a significantly higher level in the ARDS group than in control, but HMGBI expression was significantly reduced in the $\mathrm{GAL}$ group relative to the ARDS group $(p<0.05)$. In addition, HMGB1 protein expression in MLA, Vag and ATS groups were higher, when compared with corresponding expressions in the control, indicating that methyllycaconitine, vagotomy and atropine sulfate reduced GAL-induced downregulation of HMGB1 expression $(p<0.05$, Figure 3).

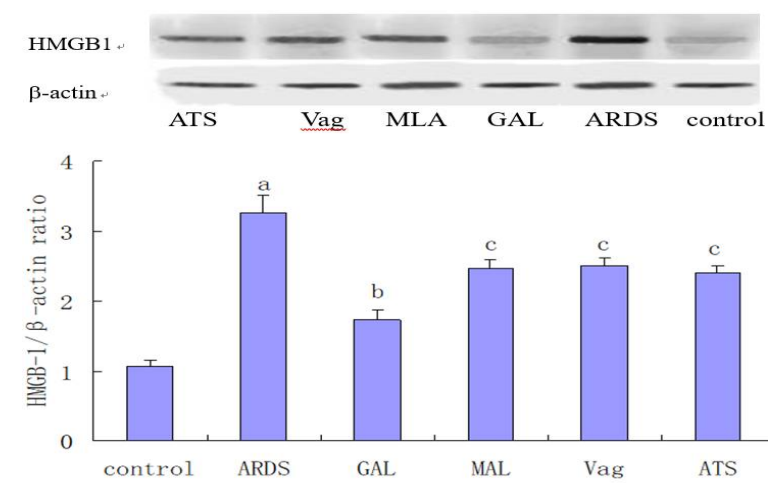

Figure 3: Expression levels of HMGB1 in pulmonary tissues of rats in the 6 groups at $12 \mathrm{~h}$. ${ }^{\mathrm{a}} \boldsymbol{p}<\mathbf{0 . 0 5}$, ARDS group vs control group; ${ }^{b} p<0.05$, GAL group vS ARDS group ; ${ }^{c} p<0.05$, MLA, Vag and ATS groups vs GAL group. Values are mean \pm SD

\section{Pro-inflammatory cytokine expressions in lungs}

In the ARDS group, TNF- $\alpha$, IL- $1 \beta$ and IL- 6 protein expressions at $4 \mathrm{~h}$ were significantly higher, relative to the control and GAL groups $(p<0.05))$. This suggests that $\mathrm{GAL}$ suppressed the expressions of TNF- $\alpha$, IL-1 $\beta$ and IL- 6 in the lungs. In addition, TNF- $\alpha$, IL$1 \beta$ and IL-6 protein expressions were significantly higher in the MLA, Vag and ATS groups than in the GAL group, suggesting that methyllycaconitine, vagotomy and atropine sulfate reduced GAL-induced downregulation of these cytokines $(p<0.05$, Figure 4).

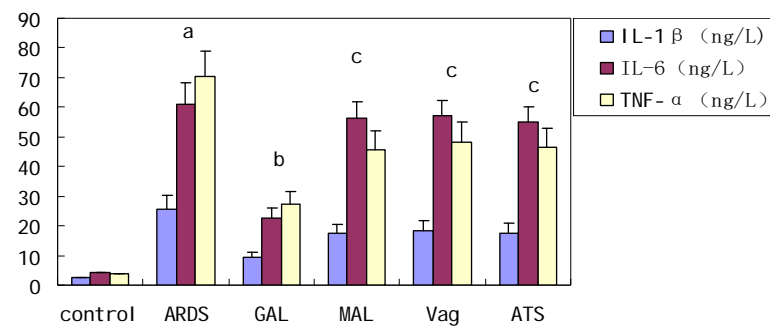

Figure 4: Effect of the various treatments on the levels of pro-inflammatory cytokines. ${ }^{a} p<0.05$, ARDS group vs control group; ${ }^{b} p<0.05$, GAL group vs ARDS group ; ${ }^{c} p<0.05$, MLA, Vag and ATS groups vs GAL group. Values are mean \pm SD

\section{Corticosterone levels}

There were no significant alterations in serum corticosterone levels amongst the various groups.

\section{DISCUSSION}

Acute acid aspiration-induced injury is a biphasic injury pattern at $1 \mathrm{~h}$ and $4 \mathrm{~h}$. The first phase results from a direct physiochemical process, while the second phase is mediated by neutrophils, and is consistent with an acute inflammatory response [13]. Elevations in TNF- $\alpha$, IL-1, and IL-6 are implicated in the etiology of ARDS. Neuronal cholinergic function is enhanced by GAL through inhibition of acetylcholinesterase (AChE), an action aided by its ability to traverse the blood brain barrier (BBB) [14]. In the present study, it was shown that GAL suppressed acute inflammatory response during the second phase of acute of acid aspirationinduced ARDS, and also suppressed the inflammation mediator HMGB1, but had no appreciable effect on serum concentrations of corticosterone. Notwithstanding the differences in animal models used, these results are in agreement with the report that GAL protects rats from LPS-provoked ALI [15]. 
The control of the neuronal cholinergic route of anti-inflammation in the efferent vagus nerve is poorly understood. However, it is known that this process is regulated in a central fashion. In the present study, it was demonstrated that GALassociated mitigation of inflammation was diminished through vagus nerve transection. Signal transmission from the vagus nerve is regulated by the activities of muscarinic receptors, as well as AChE [8]. The findings of present study have shown that atropine sulfate, an antagonist of muscarinic receptors which can also pass through BBB, eliminated GALmediated mitigation of inflammation. It has been demonstrated that impulses from the vagus nerve regulate the inflammatory processes centrally and peripherally through the nicotinic ACh receptor [16]. In this study, it was shown that the a7nicotinic receptor blocking agent methyl lycaconitine reduced GAL-induced suppression of acute inflammatory response.

\section{CONCLUSION}

The results of this study demonstrate that GAL mitigates acid aspiration-induced ARDS via inhibition of pro-inflammatory cytokine availability whose mechanism involves an interplay between $\alpha-7 n A C h R$, vagus nerve and muscarinic receptors. Thus, GAL has promising a potential for clinical application in suppressing inflammatory responses associated with ARDS.

\section{DECLARATIONS}

\section{Conflict of Interest}

No conflict of interest associated with this work

\section{Contributions of authors}

We declare that this work was done by the author(s) named in this article and all liabilities pertaining to claims relating to the content of this article will be borne by the authors. All authors read and approved the manuscript for publication. Jin Yang conceived and designed the study, Yi Yang and Yan Peng collected and analyzed the data, Yi Yang wrote the manuscript.

\section{REFERENCES}

1. Villar J, Sulemanji D, Kacmarek RM. The acute respiratory distress syndrome: incidence and mortality, has it changed? Curr Opin Crit Care 2014; 20(1): 3-9.

2. Estenssoro E, Dubin A, Laffaire E, Canales H, Sáenz G, Moseinco M, Pozo M, Gómez A, Baredes N, Jannello G,
Osatnik J. Incidence, clinical course, and outcome in 217 patients with acute respiratory distress syndrome. Crit Care Med 2002; 30(11):2450-2456.

3. Goodman RB, Pugin J, Lee JS, Matthay MA. Cytokinemediated inflammation in acute lung injury. Cytokine Growth Factor Rev 2003; 14: 523-535.

4. Huston JM. The vagus nerve and the inflammatory reflex: wandering on a new treatment paradigm for systemic inflammation and sepsis. Surg Infect 2012; 13: 187-193.

5. Bruchfeld A, Goldstein RS, Chavan S, Patel NB, RosasBallina M, Kohn N, Qureshi AR, Tracey KJ. Whole blood cytokine attenuation by cholinergic agonists ex vivo and relationship to vagus nerve activity in rheumatoid arthritis. J Intern Med 2010; 268: 94-101.

6. Akella $A$, Deshpande SB. Vagal efferent stimulation protects against Mesobuthus tamulus venom-induced acute respiratory distress syndrome in rats. Toxicon 2015; 108: 189-201

7. Tracey KJ. Physiology and immunology of the cholinergic antiinflammatory pathway. J Clin Invest 2007; 117(2): 289-296.

8. Pavlov VA, Ochani M, Gallowitsch-Puerta M, Ochani K, Huston JM, Czura CJ, Al Abed Y, Tracey KJ. Central muscarinic cholinergic regulation of the systemic inflammatory response during endotoxemia. Proc Natl Acad Sci USA 2006; 103: 5219-5223.

9. Liu ZH, Ma YF, Wu JS, Gan JX, Xu SW, Jiang GY. Effect of cholinesterase inhibitor galantamine on circulating tumor necrosis factor alpha in rats with lipopolysaccharide-induced peritonitis. Chin Med J 2010; 123: 1727-1730.

10. World Health Organization. Declaration of Helsinki. Br Med J 1996; 313(7070): 1448-1449.

11. Imanaka $H$, Shimaoka $M$, Matsuura $N$, Nishimura $M$, Ohta $N$, Kiyono $H$. Ventilator-induced lung injury is associated with neutrophil infiltration, macrophage activation, and TGF-beta $1 \mathrm{mRNA}$ upregulation in rat lungs. Anesth Analg 2001; 92:428-436.

12. Chen F, Liu Z, Wu W, Rozo C, Bowdridge S, Millman A, Van Rooijen N, Urban JF, Wynn TA, Gause WC. An essential role for TH2-type responses in limiting acute tissue damage during experimental helminth infection. Nat Med 2012; 18: 260-266.

13. Kennedy TP, Johnson KJ, Kunkel RG, Ward PA, Knight $P R$, Finch JS. Acute acid aspiration lung injury in the rat: biphasic pathogenesis. Anesth Analg 1989; 69(1): 8792.

14. Ellis JM. Cholinesterase inhibitors in the treatment of dementia. J Am Osteopath Assoc 2005; 105: 145-158.

15. Li G, Zhou CL, Zhou QS, Zou HD. Galantamine protects against lipopolysaccharide-induced acute lung injury in rats. Braz J Med Biol Res 2016; 49(2): e5008.

16. Ulloa $L$. The vagus nerve and the nicotinic antiinflammatory pathway. Nat Rev Drug Discov 2005; 4(8): 673-684. 